\title{
B. W. J. Mahy and M. H. V. Van Regenmortel (Editors-in-Chief): Encyclopedia of Virology
}

\author{
Third Edition, 2008, Elsevier-Academic Press, 1485 USD (1190 €)
}

\author{
Hans-Dieter Klenk
}

(C) Springer-Verlag 2009

If you are asked about allexivirus, marnavirus or Taura Syndrome virus and you do not know what to answer, consult the last edition of the Encyclopedia of Virology which is the largest reference source of virological information available to date. Whereas even the most comprehensive textbooks focus primarily on viruses of medical importance and interest, the Encyclopedia includes animal, insect, plant, and bacterial viruses with equal emphasis. There are more than 400 articles addressing general topics as well as specific viruses and virus families. Each entry describing a particular virus contains the most recent taxonomic information as provided by the 8th Report of the International Committee on Taxonomy of Viruses. General aspects are illustrated by articles on subjects ranging from the evolution and phylogeny of viruses and the history of virology to innate and adaptive immunity, to disease surveillance, virus vectors in gene therapy and antivirals. Many chapters emphasize the economic importance of virus infections of major crops and other plants and new approaches to control these diseases. A large part of the presented information is new and has been derived from the research of the last couple of years. This is reflected by the increase in the size of the Encyclopedia which comprises now five volumes as compared to the three volumes of the first and the second editions.

More than 600 scientists have contributed to these 5 volumes. It is therefore not surprising that sometimes several articles are devoted to one theme. In general, however, there is little overlap. For instance, there are three entries on AIDS (disease manifestation, global epidemiology, vaccine development), four entries on HIV (antiviral agents, molecular biology, origin, pathogenesis), and another three entries on SIV. Thus, each of these contributions illuminates the same subject from a different point of view and with the eyes of a different viewer. In spite of the large number of authors all chapters are surprisingly homogeneous in format and style. This is an impressive editorial accomplishment. The text is complemented by very informative illustrations, and each article has a Further Reading section at the end containing a list of reviews on the particular subject. It may be deplored that references to original publications are missing, but as a concession to space limitations this was probably inevitable. With the huge amount of information presented, it is essential for the reader to easily find access to what he is looking for. A cross-reference section at the end of each article directs to other entries on related topics. Furthermore and most importantly, the Encyclopedia has an index listing about 25,000 items, more than any other comparable publication.

The Encyclopedia is highly recommended as a rapid source of information and a teaching aid to all virologists and to scientists working in related fields. It should be present in every virus laboratory to make it also available to graduate students and postdocs who otherwise might be deterred by the high prize from buying their own copy.

H.-D. Klenk ( $\square)$

Institute of Virology, Marburg, Germany

e-mail: klenk@staff.uni-marburg.de 